\title{
Study to detect incidence of carbapenemase among Gram negative clinical isolates from tertiary care hospital
}

\author{
Balan K, Sireesha P, Setty CR
}

\begin{abstract}
Purpose:To study incidence of carbapenemases in teritary care hospital among gram negative clinical isolates of both Enterobacteriaceae and nonenterobactiaceae(nonferementers).

Materials and Methods: A totalof 200 gram negative isolates were subjected to various phenotypic methods for carbapenemase detection along with minimum inhibitory concentration(MIC) for Imipenem and Meropenem by agar dilution technique.The phenotypic methods testedlike Double disc synergy test(DDST), Combined disc test (CDT), Modified hodge test(MHT).

Results: Of 200 isolates, 114 were members of famiy enterobacteriaceae and 86 were nonferementers. Out of 114 , 71 were E.coli, 32 Klebsiella spp, 08 enterobacter and 03 citrobacter. Out of 86 nonfermenters 55 were pseudomonas spp and 31 were acinetobacter spp.Of the 200 isolates tested by DDST, 06 showed distortion of the one towards the EDTA disc.In CDT test 30 isolates showed an increase of $>7 \mathrm{~mm}$ zone of inhibition around the imipenem+EDTA disc in comparison to imipenem alone. Similarly 18 isolates had an increase of $>7 \mathrm{~mm}$ around the ceftazidime+EDTA in comparison to ceftazidime alone. Only one Klebsiella isolate was found to be positive with MHT, where a clover leaf shaped zone of inhibition was noted.Of 200 isolates, 167 were found to have an MIC of < $2 \hat{A} \mu \mathrm{g} / \mathrm{ml}, 25 \mathrm{had}$ an MIC value of $2 \hat{A} \mu \mathrm{g} / \mathrm{ml}, 07 \mathrm{had}$ an MIC of $4 \hat{A} \mu \mathrm{g} / \mathrm{ml}$ and 01 had an MIC of $8 \hat{A} \mu \mathrm{g} / \mathrm{ml}$ against imipenem. For meropenem, 170 isolates had an MIC of $<2 \hat{A} \mu \mathrm{g} / \mathrm{ml}, 22$ had an MIC of $2 \hat{A} \mu \mathrm{g} / \mathrm{ml}, 07 \mathrm{had}$ an MIC of $4 \hat{A} \mu \mathrm{g} / \mathrm{ml}$ and 01 had an MIC of $8 \hat{A} \mu \mathrm{g} / \mathrm{ml}$. The strain, Klebsiella spp, showed higher MIC $8 \hat{A} \mu \mathrm{g} / \mathrm{ml}$ for both imipenem and meropenem.

Conclusions: Different phenotypic methods for detection of these carbapenemases are available, but controversies exist regarding the choice of optimal laboratory method. Molecular methods though expensive are the confirmatory tests
\end{abstract}

\section{Introduction}

Resistant bacteria are emerging worldwide both in community and hospital settings. There are many reports of increase in the incidence of noscomial infections by multidrug resistant organisms. ${ }^{(1)}$

Bacteria have developed several mechanisms of resistance against various antibiotics. Synthesis of drug inactivating enzymes like $\beta$ lactamases, which hydrolyse the $\beta$ lactam antibiotics, decreased target susceptibility by target alteration, development of efflux system and modification of diffusion barrier, altered metabolic activity are the main mechanisms of drug resistance. Many studies were done on $\beta$ lactam group of antibiotics and $\beta$ lactamases. ${ }^{(2)}$

In the past, multidrug resistant gram negative bacterial infections were successfully treated with penicillin group of antibiotics like carbenicillin, ticarcillin, piperacillin etc. Among all $\beta$ lactams, carbapenem group of drugs have maximum antimicrobial spectrum. This spectrum is due to their high affinity for penicillin binding protein 2 , good stability against most serine based $\beta$ lactamases and excellent outer membrane permeability. Carbapenems are among the few backup agents for multidrug resistant gram negative pathogens. However, increasing usage of carbapenems has resulted in development of resistance to this group of drugs as well. ${ }^{(3)}$

\section{Materials and methods}

Two hundred gram negative isolates from various clinical specimens like pus 129, urine 52, pleural fluid 03, sputum 07, endotracheal secretions 07 and blood 02 were taken for the study. Of these 200 samples, 147 were from in patients from wards and 53 were from ICU (Table 1) Of the 200 isolates, 114 were members of the family enterobacteriaceae and 86isolates were nonfermenters. Out of these 114, 71 were E.coli, 32 Klebsiella spp, 08 enterobacter spp and 03 citrobacter spp. Out of the 86 nonfermenters, 55

were pseudomonas spp and 31 acinetobacter spp These isolates were subjected to various phenotypic methods of carbapenemase detection along with MIC determination for imipenem and meropenem. The phenotypic methods tested were double disc synergy test, combined disc test and Modified Hodge test. The MIC was determined by agar dilution technique. 


\section{DOUBLE DISC SYNERGY TEST (DDST) ${ }^{(4)}$}

\section{Phenotypic Methods}

After purity check, isolated colonies were inoculated into peptone water and kept in incubator at $37^{\circ} \mathrm{C}$ for 2 hours to get a turbidity to match $0.5 \mathrm{Mc}$ Farland tube. The test organism inoculum was spread as a lawn onto Muller Hinton agar (MHA) plate using a sterile cotton swab. A $6 \mathrm{~mm}$ in diameter Whatman No 1 filter paper disc with $10 \mu \mathrm{l}$ of EDTA $(\approx 1900 \mu \mathrm{g}$ of disodium salt dehydrate $)$ solution was placed at center of the plate. On either sides of this EDTA disc, at a distance of $10 \mathrm{~mm}$ from the edge to edge, an imipenem $(10 \mu \mathrm{g})$ and a ceftazidime $(30 \mu \mathrm{g})$ discs were placed. The inoculated plates were incubated at $35^{\circ} \mathrm{C}$ in the incubator for 18-24 hours. Presence of any distortion in the zone of inhibition or any indentation towards the EDTA disc was interpreted as positive.

\section{COMBINED DISC TEST (CDT) ${ }^{(4)}$}

In this test after spreading the test inoculum (0.5 Mc Farland turbidity) on to MHA plate, two imipenem $(10 \mu \mathrm{g})$ discs and two ceftazidime $(30 \mu \mathrm{g})$ discs were placed onto one of the imipenem and ceftazidime discs, $10 \mu \mathrm{l}$ of EDTA solution was added. The plates were incubated at $35^{\circ} \mathrm{C}$ for $18-24$ hours. The zone of inhibition between the imipenem and imipenem + EDTA and ceftazidime and ceftazidime + EDTA discs were compared. Difference in the zone diameter of $\geq 7 \mathrm{~mm}$ was interpreted as positive for MBL production.

\section{MODIFIED HODGE TEST (MHT) ${ }^{(5)}$}

The ATCC E.coli 25922 at a turbidity equivalent to that of $0.5 \mathrm{Mc}$ Farland was inoculated onto the MHA plate. After brief drying for five minutes, a meropenem $(10 \mu \mathrm{g})$ disc was placed at the centre of the plate. The test strain was heavily streaked from the edge of the meropenem disc to the periphery of the plate. Four isolates were inoculated in one plated at $90^{\circ}$ to each other and the plates were incubated at $35^{\circ} \mathrm{C}$ for $18-24$ hours. Presence of a clover leaf type of zone of inhibition near the test organism was interpreted as positive for carbapenem hydrolyzing enzymes, as per the CLSI guidelines.

\section{MINIMUM INHIBITORY CONCENTRATION (MIC)}

MIC to imipenem and meropenem was done by agar dilution technique. Commercial preparation of imipenem (500 mg) (Manufacturers: M/S. Zeiss pharma (P) Ltd) and meropenem (500 mg) (Astra Zeneca pharma India Ltd) were taken for the study. ATCC Pseudomonas aeruginosa 27853 was used as control strain MIC values for both imipenem and meropenem were interpreted as follows: $\leq 1 \mu \mathrm{g} / \mathrm{ml}$ as sensitive, $2 \mu \mathrm{g} / \mathrm{ml}$ as intermediate and $\geq 4 \mu \mathrm{g} / \mathrm{ml}$ as resistant as per CLSI guidelines. ${ }^{(6)}$

\section{Results}

Of these 200 samples, 114 yielded organisms belonging to enterobacteriaceae and 86 yielded growth of nonfermenters. Out of 114 members of enterobacteriaceae family, 71 were E.coli, 32 klebsiella spp, 08 enterobacter spp and 03 citrobacter spp. Out of 86 nonfermenters, 55 were pseudomonas spp and 31 were acinetobacter spp.

Table 1: Shows the breakup of organisms isolated from patient in to various wards

\begin{tabular}{|l|c|c|c|c|c|c|c|}
\hline \multicolumn{1}{|c|}{ Organism } & ICU & Surgery & Ortho & Medicine & Gynae & Paed & Total \\
\hline E.coli & 16 & 24 & 1 & 15 & 13 & 01 & 71 \\
\hline Pseudomonas spp & 17 & 25 & 05 & 05 & 03 & - & 55 \\
\hline Klebsiella spp & 12 & 08 & 03 & 07 & 01 & 01 & 32 \\
\hline Acinetobacter spp & 08 & 18 & - & 03 & 02 & - & 31 \\
\hline Enterobacter spp & - & 04 & 02 & 02 & - & - & 08 \\
\hline Citrobacter spp & - & 01 & - & 02 & - & - & 03 \\
\hline Total & 53 & 80 & 11 & 94 & 19 & 02 & 200 \\
\hline
\end{tabular}

Of the 200 isolates tested by DDST, 06 showed distortion of the zone towards the EDTA disc. Of these 06 isolates 02 were E.coli, 02 acinetobacter spp, 01 klebsiella spp and 01 pseudomonas spp.

In the combined disc test, 30 isolates showed an increase of $>7 \mathrm{~mm}$ zone of inhibition around the imipenem+ EDTA disc in comparison to imipenem alone. Similarly 18 isolates had an increase of $>7 \mathrm{~mm}$ around the ceftazidime+ EDTA disc in comparison to ceftazidime alone. Eleven isolates were positive (showed $>7 \mathrm{~mm}$ difference in zone size) with both imipenem+EDTA and ceftazidime+ EDTA in comparison to imipenem and ceftazidime alone. Only one klebsiella isolate was found to be positive with modified hodge test, where a clover leaf shaped zone of inhibition was noted around the isolate 
Table 2: Comparison of various phenotypic tests for detection of carbapenemases

\begin{tabular}{|ll|c|c|}
\hline & Methods & No of strains & Percentage \\
\hline & DDST & 06 & 03 \\
\hline & CDT & 30 & 15 \\
a. & Imipenem & 18 & 09 \\
b. & Ceftazidime & 01 & 0.5 \\
\hline & MHT & & \\
\hline
\end{tabular}

MIC determination for imipenem and meropenem was done by agar dilution technique; where in a range of $2 \mu \mathrm{g} / \mathrm{ml}$ to $16 \mu \mathrm{g} / \mathrm{ml}$ of the drug concentrations were as covered. Of these 200 isolates, 167 isolates were found to have an MIC of $<2 \mu \mathrm{g} / \mathrm{ml}, 25 \mathrm{had}$ an MIC value of $2 \mu \mathrm{g} / \mathrm{ml}, 07 \mathrm{had}$ an MIC of $4 \mu \mathrm{g} / \mathrm{ml}$ and $01 \mathrm{had}$ an MIC of $8 \mu \mathrm{g} / \mathrm{ml}$ against imipenem. For meropenem, 170 isolates had an MIC of $<2 \mu \mathrm{g} / \mathrm{ml}, 22$ had an MIC of $2 \mu \mathrm{g} / \mathrm{ml}, 07 \mathrm{had}$ an MIC of $4 \mu \mathrm{g} / \mathrm{ml}$ and $01 \mathrm{had}$ an MIC of $8 \mu \mathrm{g} / \mathrm{ml}$. The strain, Klebsiella spp, showed higher MIC $8 \mu \mathrm{g} / \mathrm{ml}$ for both imipenem and meropenem.

Table 3: Shows minimum inhibitory concentration of imipenem and meropenem by agar dilution technique.

\begin{tabular}{|c|c|c|c|c|}
\hline \multirow{2}{*}{ Drugs tested } & \multicolumn{4}{|c|}{ Minimum Inhibitory Concentration in $\mu \mathrm{g} / \mathrm{ml}$} \\
\cline { 2 - 5 } & $<2$ & 02 & 04 & 08 \\
\hline Imipenem & 167 & 25 & 07 & 01 \\
\hline Meropenem & 170 & 22 & 07 & 01 \\
\hline
\end{tabular}

\section{Discussion}

The discovery and development of antibiotics is one of the greatest advances in modern medicine. Carbapenems are $\beta$ lactam antibiotics presently considered as potent agents to target multidrug resistant gram negative infections, due to the stability of these agents against many $\beta$ lactamases.

The prevalence of carbapenemase among gram negative bacilli varies greatly from country to country and among different institutions within the country. Carbapenemase production is only one mechanism of resistance and other mechanisms also exist for carbapenem resistance like porin loss, efflux of drug and target alteration. However, it is well known that multiple mechanisms of resistance and enzyme production can coexist in a single organism.

Carbapenem hydrolyzing enzymes are most commonly seen in nonfermenter gram negative organisms (non enterobacteriaceae) ie. Pseudomonas and acinetobacter. However, in the recent years there is an increasing incidence of these enzymes in enterobacteriaceae family as well. In Enterobacteriaceae family carbapenemases are more common encountered in lactose-fermenters (e.g. K.pneumoniae and E.coli) than in non-lactose fermenters (e.g. Serratia marcescens and some Enterobacter spp).

The present study was under taken to compare the different phenotypic methods for carbapenemase detection in nonfermenters and enterobacteriaceae family from various clinical isolates in our hospital.

Though CLSI does not advocate the use of the modified Hodge test for the detection of carbapenemase production in nonfermenting Gram-negative bacilli, several authors have found the modified Hodge test using imipenem as a useful screening test for carbapenemase production. In the present study we screened all the isolates with three different phenotypic methods to detect both MBLs and other carbapenamase.

Initially carbapenem resistant strain were observed in P.aeruginosa and in no time they disseminated to different parts of the world. ${ }^{(7)}$ Two studies by Marchiaro et al ${ }^{(8)}$ and Toleman et al ${ }^{(9)}$ reported the prevalence of MBL to be $43.9 \%$ and $39.1 \%$ in P.aeruginosa. In the year 2010, in India, a study done by Manoharan et al ${ }^{(10)}$ quoted the prevalence of MBLs in P.aeruginosa to be as high as $65 \%$. In acinetobacter spp, in a study by Manikal et al, ${ }^{(11)}$ in the year 2000 in New York hospitals, the MBLs prevalence was found to be $50 \%$.

Later in one Indian study by Taneja et al, in 2003 the prevalence of MBL in acinetobacter was found to be $20 \%$. There are very few studies for carbapenemase detection in acinetobacters when compared to P.aeruginosa. ${ }^{(7)}$ In the present study the prevalence of MBL in P.aeruginosa is $15 \%$ which correlates with two studies by Hemalatha et al ${ }^{(12)}$ and Navaneeth et al ${ }^{(13)}$ where the prevalence was $16 \%$ and $12 \%$. Prevalence of 
MBL in acinetobacter spp in present study is $10.5 \%$ while a study by Glandstone $e a^{\left(l^{(14)}\right.}$ in 2005 was $14.2 \%$ because in the Indian scenario production of MBL may not play a major role in carbapenem resistance among acinetobacters spp. ${ }^{(15)}$

In DDST 6 isolates (3\%) were positive of which 02 were E.coli, 02 acinetobacters spp, 01 Klebsiella spp and 01 pseudomonas spp. In the present study the distance between the EDTA and the other two antibiotic discs was taken as $10 \mathrm{~mm}$. In literature many authors have chosen $10 \mathrm{~mm}$ distance between the discs as it was found to be more sensitive compare to other distance. The study by Galani et al ${ }^{(4)}$ observed that $10 \mathrm{~mm}$ distance between imipenem and EDTA disc shows sensitivity (100\%) and specificity (91.9\%). There are very few studies in literature to compare the sensitivity of imipenem and ceftazidme in detecting MBLs by DDST, two such studies are by Mendiratta $e t a l^{(4)}$ and Hemalatha $e t a I^{(12)}$ where they found ceftazidime to be a better disc in comparision to imipenem in detecting the MBLs. In the present study however, study we did not find any difference between imipenem and ceftazidme.

Many chelating agents were used in testing MBLs, however most commonly used is EDTA, but it has a direct bactericidal effect on test strain which can give rise to false positive. ${ }^{(15)}$ A study by Galani et $a l,{ }^{(17)}$ shows the sensitivity of DDST varies from 44 to $67 \%$. However EDTA disc were more sensitive than SMA disc for detection of MBL production in pseudomonas, whereas SMA was better for acinetobacter spp. In the present study we used only EDTA as a chelating agent.

In CDT, 30 isolates (15\%) were found to be positive with imipenem + EDTA and 18 isolates (09\%) with ceftazidime + EDTA, in detecting MBLs. Similar to our study, Sakshi et al ${ }^{(18)}$ in 2009 found a sensitivity of $95.2 \%$ using IPM+EDTA, in comparison to $38.1 \%$ using ceftazidime +EDTA. In the same year Manoharan et $a l^{(10)}$ also found a better sensitivity of $93.3 \%$ with imipenem +EDTA in detecting MBL as compared to EDTA+ ceftazidime. In contrast to our study, studies by Hemalatha et $a l^{(12)}$ and Arakawa et $a l^{(19)}$ found ceftazidime to be a better agent in detecting MBL than imipenem, however, in our study imipenem was found to be a better choice for detection of MBL production by CDT.

So CDT is more sensitive screening method in detecting MBL. ${ }^{(7)}$ However, though different phenotypic methods have been described for a long time there are no standard guidelines for screening of carbapenemase. (15) CLSI has recommended MHT for detection of carbapenemase activity in enterobacteriaceae, especially for KPC in klebsiella spp, but not in nonfermenters. However, we performed this test on all isolates to see for any co-existence of different types of enzymes. In our study, only one out $(2.8 \%)$ of 35 klebsiella isolates was found to be positive with MDT, where a clover shaped zone of inhibition was noted around the isolate.

In the present study, 167 (83.5\%) out of 200 the isolates were sensitive to imipenem, where the MIC was $<2$ $\mu \mathrm{g} / \mathrm{ml}, 25$ isolates (12.5\%) were intermediate sensitive with an MIC of $2 \mu \mathrm{g} / \mathrm{ml}$ and 08 isolates (04\%) were found to be resistant with 07 having an MIC of $4 \mu \mathrm{g} / \mathrm{ml}$ and one with an MIC of $8 \mu \mathrm{g} / \mathrm{ml}$.

In 2008 Agarwal et al ${ }^{(20)}$ observed that $77.01 \%$ of strains for imipenem were having MIC as low as $\leq 2 \mu \mathrm{g} / \mathrm{ml}$, while $1.15 \%$ of strains were having MIC as high as $1024 \mu \mathrm{g} / \mathrm{ml}$. Such a high MIC was not observed in present study.

MICs against meropenem was as follows $85 \%$ strains had MIC of $<2 \mu \mathrm{g} / \mathrm{ml}, 11 \%$ show MIC of 2 $\mu \mathrm{g} / \mathrm{ml}, 3.5 \%$ showed $4 \mu \mathrm{g} / \mathrm{ml}$ and $0.5 \%$ showed MIC of $8 \mu \mathrm{g} / \mathrm{ml}$. A study done by Hemalatha et al observed that MIC ranges from 8-128 $\mu \mathrm{g} / \mathrm{ml}$ but in our study only one isolate showed high MIC of $8 \mu \mathrm{g} / \mathrm{ml}$. Four isolates which shows high MIC of $4 \mu \mathrm{g} / \mathrm{ml}$ did not exhibit zone size potentiation with EDTA impregnated antibiotics discs which suggests other mechanism of resistance like impaired permeability or active efflux pump associated with overproduction of endogenous $\beta$ lactamase being responsible for this high MIC.

Of the 200 strains, eight isolates showed an MIC of $>4 \mu \mathrm{g} / \mathrm{ml}$ for both imipenem and meropenem. When these isolates were compared to various phenotypic tests (DDST, CDT, MHT), seven strains were positive for CDT, two strains were found to be positive for DDST and one isolate which is klebsiella spp. positive for all three test like DDST, CDT and MHT. This shows CDT can detect more number of isolates than DDST.

\section{Conclusion}

Penems are $\beta$ lactam antibiotics that are the last line of drugs for treating gram negative infections. However various mechanisms of resistance have evolved for these antibiotics too due to their extensive use throughout the world. Different phenotypic methods for detection of these carbapenemases are available, but controversies exist regarding the choice of optimal laboratory method. Molecular methods though expensive are the confirmatory tests. 


\section{References}

[1] Kohler T, Epp SF, Curty LK, Pechere JC. Characterisation of Mex T, the regulator of the Mex E - Mex F - Opr N multidrug efflux system of Pseudomonas aeruginosa. J Bacteriol. 1999;20:6300 - 5

[2] Laraki N, Franceschini , Rossolini GM, Santucci P, Meunier C, de Paucv E, et al. Biochemical characterization of Pseudomonas aeruginosa 101/1477 metallo $\beta$ lactamase IMP - 1 produced by Escherichia coli. Antimicrob Agents Chemother 1999;4:902-65.

[3] Livermore DM.Carbapenemases.J Antimicrob Chemother 1992; 6:609-6

[4] Galani I,Rekatsina PD, Hatzaki D et al. Evaluation of different laboratory tests for detection of metallo- $\beta$-lactamase production in Enterobacteriaceae. J Antimicrob Chemother 2008;25:1-6

[5] National Committee for Clinical Laboratory Standards. Performance standards for antimicrobial susceptibility testing. Twentyfirst informational supplement. M100-S21 NCCLS, 2002.Wayne P.A.

[6] Hsuch PR, Ko WC, Wa JJ, Lu JJ et al. Consensus Statement on the Adherence to Clinical and Laboratory Standards Institute (CLSI) Antimicrobial Susceptibility Testing Guidelines (CLSI-2009 and CLSI-2010 update) for Enterobacteriaceae in Clinical Microbiology in Taiwan. J Micrbol Immunol Infect 2010; 43:452-55

[7] Arora S, Saha S, Bal M. Imipenem Resistant among multidrug Resistant clinical strain in Urinary infection from Kolkata. Indian J Med Res 2007; 125:689-91

[8] Machiaro P, Mussi MA, Ballerini V, Pasteran F, Viale AM, Vila AJ, et al. Sensitive EDTA based microbiological assay for detection of metallo $\beta$ lactamase in nonfermentative Gram negative bacteria. J Clin Microb 2005; 43:5648-52

[9] Toleman MA, Biedenbach D, Bennet DMC, Jones RN, Walsh TR. Italian metallo $\beta$ lactamase: a national problem? Report from the SENTRY Antimicrobial Surveillance Program. J Antimicrob Chemother 2005;55:61-70.

[10] Manoharan A, Chatterjee S, Mathur D, SARI Study Group. Detection and characterization of metallo $\beta$ lactamases producing Pseudomonas aeruginosa. Indian J Microbl 2010; 28:241-4.

[11] Manikal UM, Landman D, Saurina G, Oydna E, Lal H, Quale J. Endemic carbapenem resistant Acinetobacter species in Brooklyn. Newyork:Citywide prevalence,inter-institutional spread and relation to antbiotic usuage. Clin Infect Dis 2000; $31: 101$ 6 .

[12] Hemalatha N, Uma Sekar, Kamat V. Detection of metallo $\beta$ lactamase producing Pseudomonas aeruginosa in hospitalsed patient. Indian J Med Res 2005; 122:148-52.

[13] Navaneeth BV, Sridaran D, Sahar D, Belwadi MRS. A preliminary study on metallo $\beta$ lactamase producing Pseudomonas aeruginosa in hospitalised patient. Indian J Med Res 2002; 116:264-7.

[14] Gladstone P, Rajendran P, Bramadthan KN. Inicdence of carbapenem resistant nonfermentating Gram negative bacilli from patients with Respiratory infection in intensive care units. Indian J Med Microbiol 2005; 25:189-91.

[15] Noyal MIC, Menezer GA, Harish BN, Sujatha, Parija SC. Simple screening test for detection Carbapenemases in clinical isolates of nonfermentative Gram negative bacteria. Indian J Med Res 2009;129:707-12.

[16] Mendiratta DK, Deotale V, Narang P. Metallo $\beta$ - lactamase producing Pseudomonas aeruginosa in hospitalised patient from rural area. Indian J Med Res 2005; 121:701-3.

[17] Kim SY, Hong SG, Moland ES, Thomas S. Convenient test using a combination of chelating agents for detection of Metallo- $\beta$ lactamases in the clinical laboratory. J Clin Microbiol 2007; 45:2798-801.

[18] Sakshi P, Barua MST, Thaurkral SS. Comparitive evaluaton of Phenotypic test for identifcaton of metallo $\beta$ - lactamase producing clinical isolates of Pseudomonas aeruginosa. Indian J Med Res 2009; 129:713-15.

[19] Arakawa Y, Shibata N, Shibayama K, Kurokawa H, Yagi T, Fujiwara H, et al. Convenient test for screening metallo $\beta$ lactamases producing Gram negative bacteria by using thiol compounds. J Clin Microbiol 2000; 38:40-3

[20] Lee K, Lim YS, Yong D, Yum JH, Chong Y. Evaluation of Modified Hodge Test and the Imipenem- EDTA Double Disk Synergy Test for Differentiating Metallo- $\beta$ - lactamase producing of Pseudomonas spp. and species and Acinetobacter spp. J Clin Microbiol 2003;41:4623-29. 